\title{
Food Diversification Levels and Nutrients in Tuberculosis Patients With and Without Diabetes Mellitus
}

\author{
Guo Yumei, a, Wang Qiuzhen ${ }^{1, b^{*}}$, Liu Yufeng ${ }^{2, c}$, Ma Aiguo ${ }^{1, d}$, Zhao Yanxu $^{2, e}$, \\ Zou Yue ${ }^{2, f}$, Sun Limei ${ }^{2, g}$, Tian Hong ${ }^{2, h}$, Jiang Guofeng ${ }^{2, i}$, Lin Ruihan ${ }^{1, j}$, Wang \\ Yin $^{1, k}$, Dou Xiaojuan ${ }^{1,1}$ \\ ${ }^{1}$ Nutrition Institute, Qingdao University, Qingdao, China \\ ${ }^{2}$ Qingdao Chest Hospital, Qingdao, China \\ a782819821@163.com, ${ }^{\mathrm{b}}$ kevin_1971@126.com, 'Y Yufengliu@126.com, ${ }^{\mathrm{d}} \mathrm{ma91503@126.com,}{ }^{\mathrm{e}} \mathrm{Yan}$ \\ xuzhao@126.com, ${ }^{\mathrm{f} Y} Y u e z o u @ 126 . c o m,{ }^{9}$ Limeisun@126.com, ${ }^{\mathrm{h}}$ Hongtian@126.com, 'Guofengjiang

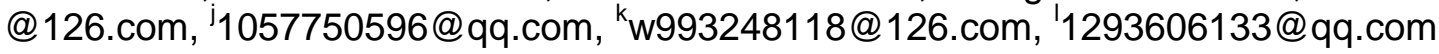

Keywords: Food Diversification Levels, Nutrients Intake, tuberculosis (TB), Tuberculosis with diabetes mellitus (TB-DM)

\begin{abstract}
We aimed to investigate the food diversification levels and nutrients intake of tuberculosis patients with and without diabetes mellitus. Participants were diagnosed and treated at Qingdao Chest Hospital between April and August 2015. A total of 131 patients were eligible in the study and 50 of them were suffering from tuberculosis (TB) and diabetes mellitus (DM) simultaneously, 81 were TB only. Food types were detected by the investigation of dietary frequency, and the dietary intake was collected by the method of recall 24 hour food intake. This survey found that most of patients in TB-DM patients group reached dietary diversification. The types of food intake results show that beans and milk intake in TB patients group were lower than that in TB-DM patients group. Our study showed that daily intake of energy and most nutrients of TB-DM patients in our study were significantly lower than the Recommended Nutrient Intakes (RNI).
\end{abstract}

\section{Introduction}

It has been attracted much number of attention that the complicating epidemics of pulmonary tuberculosis (TB) and diabetes mellitus (DM) and their effect on human health[1]. The prevalence of diabetes in China has an incredible increase. It was reported that the age standardized prevalence of diabetes mellitus and impaired glucose tolerance (IGT) was $9.7 \%$ and $15.5 \%$, separately[2]. At the same time, China has a high burden, accounting for approximately seventeen percent of the world's TB burden. There are estimated 1.5 million new cases and approximately two hundred and seventy thousand deaths due to tuberculosis every year in China. A large meta-analysis reported that compared with non-diabetic controls, the DM patients were 3.1 times to have TB[3] and it has been assessed that the TB risk attributable to DM was between 15\% and 25\%[4] Similar findings with risk ratios of 3.47 and 3.80 were shown in two cohort studies from Korea and UK[5, 6]. Therefore, the double burden and combined prevalence of tuberculosis and diabetes mellitus portray a huge health threat, making treatment failure more frequently, resulting in more community-acquired tuberculosis[7]. Nutrient consumption is a major feature of TB, and causing TB patients with different degrees of malnutrition[8], which will enhance the injury of patient's immune function. It also delayed the healing of the illness and even lead to the progression and deterioration of active pulmonary tuberculosis disease [9]. Therefore, rational nutrition guidance plays an essential role in TB patients' treatment and rehabilitation. The control of blood glucose in patients with diabetes mellitus has a closely relationship with daily diet, and patients with diabetes mellitus need to implement nutrition therapy to control the energy intake as long as their lives. When pulmonary tuberculosis patients combined with diabetes, it makes it harder for medical workers to get the two kinds of mutual conflicts of the collocation to rationalize, so as to enhance the recovery of the disease. 
In this study, we assess the dietary patterns and nutrient intake level among TB patients with and without DM to evaluate nutritional status and provide a scientific basis to direct their diet.

\section{Methods}

Design and instrument. This is a cross-sectional study. The recruited participants were patients diagnosed and treated at Qingdao Chest Hospital between April 2015 and August 2015. A total of 131 patients were eligible in the study and 50 of them were suffering from both TB and DM, 81 of them were TB only. A two -part questionnaire including demographic data for the first part, food frequency table and 24-hour dietary recall survey for the second part was designed.

Population and participant selection. During the study period, all eligible patients were: 1) Diagnosed with tuberculosis. The diagnosis of TB was made within the TB prevention and control system in China, where clinical symptoms, sputum smear and chest $\mathrm{x}$-ray were the principal component. 2) Diagnosis of DM was based on WHO criteria for the classification of glucose tolerance based on fasting plasma glucose (FPG). 3) Over 16 years of age. 4) Who were sufficiently able to read and write Chinese characters and have the ability to complete the questionnaire by themselves. 5) Voluntarily participating the investigation. Patients were removed from this study if they were: 1 ) With other serious physical and mental disorders. 2) Unconsciousness (Confusion) and cannot take care of themselves in daily life. 3) Reluctant to participate in this study.

Measures. The demographic information of the participants was collected: gender, age, education level, marital status, occupation, income, smoking and drinking history and current situation, etc. Data collecting was conducted through reading medical records and face-to-face interview.

Anthropometric measurements containing height and weight were measured by well-trained researcher by standard procedure. Height and weight were measured by trained investigators using standard procedure. Body mass index $\left(\mathrm{BMI}, \mathrm{kg} / \mathrm{m}^{2}\right)$ was calculated by using the formula: $\mathrm{BMI}=$ Weight $(\mathrm{kg}) / \operatorname{Height}^{2}\left(\mathrm{~m}^{2}\right)$, and the cut-off value for Chinese population was used.

It has four frequency units (times / day, times / week, times / month and times / year) in food frequency questionnaire. With the exception of the fats and oils intake in family unit to investigate food frequency, others were studied by individuals. According to the Chinese Residents Balanced Diet Pagoda[10], our daily food were divided into 9 major categories: grains, vegetables, fruits, poultry meat, fish and shrimp, milk and dairy products, eggs, beans and its products, fats and oils. In the light of the food frequency table, the kinds of food consumed every week (excluding food consumption frequency and quantity) was used to cumulative calculate Dietary Diversity Score (DDS). The study-subject can get 1 point if he eats only one kind of food for a week, and he can get less than 9 points in total. Carbonated drinks, alcoholic drinks, coffee and candy do not take part in scoring. If the patient's score is more than 7 points, we can say that his diet has reached the dietary diversification[11].

Thanks to 24-hour dietary recall table, we collect the type, weight and composition of all intake food (excluding condiments) in the survey. According to the type and quantity of intake food, we used the nutrition calculator module of family dietitian- nutrition expert system veil.1 (Beta) to calculate the participants' daily intake of energy and various nutrients.

The Ethics Committee of Qingdao Disease Prevention and Control Centre approved the present study, and written informed consent was obtained from each subject. The study was registered in the Chinese Clinical Trial Registry (No.ChiCTR-IPR-15006395).

Data analysis. SPSS Software program version17.0 was used for statistical analysis. All date are presented as means ( \pm standard deviation [SD]) for quantitative variables and counts and percentages (\%) for qualitative variables, which were calculated to describe the sample. Patient demographic characteristics , the various score of DDS and Energy and other nutrient intake level were compared using chi-squared test for qualitative variables and an independent sample t-test (normality distributed data) or Mann-Whitney U test (no normality distributed data) for quantitative variables. A p-value of $<0.05$ was considered to signify statistical significance. 


\section{Results and discussion}

Social demographic, anthropometric and lipid characteristics. Totally, 131 patients were eligible in the study and 50 of them were suffering from both TB and DM (TB-DM patients group), 81 of them were TB only (TB patients group). The demographic, anthropometric characteristics data of the study participants were shown in Table 1 . The 81 TB cases included in the study had a mean age of 33.41 years and $61.7 \%$ were male. In TB-DM group, $66 \%$ of the patients were male, with a higher age than TB group $(P=0.000)$. Patients in TB patients group were more likely to had a lower BMI $(P=0.001)$, had a higher Education level, had less average monthly income and were more often single compared with the group with TB-DM. In the TB patients group, the smoker take a percent of $63 \%$ and this percentage was obviously higher than that in TB-DM patients group $(P=0.005)$.

As we all know, the elderly population had a higher incidence of diabetes. The age data from this study indicated that patients of TB-DM patients group older than TB patients group. This result indicated that the symptoms of pulmonary tuberculosis had occurred after diabetes in the TB-DM patients of our study. In our study; BMI in TB-DM patients group was higher than that in TB patients group. A study showed that the proportion of overweight (BMI: $25.0-29.9 \mathrm{~kg} / \mathrm{m}^{2}$ ) and obesity (BMI $\geq 30.0 \mathrm{~kg} / \mathrm{m}^{2}$ ) in DM patients was higher than that in patients without DM. But the association for BMI is complex due to co-morbid DM and TB. Overweight and obesity is associated with increasing the risk of DM, and is defense against TB disease [12]. Regarding education, the great majority of the participants had accomplish elementary education, which makes sure the accuracy of patients' comprehensions of the questionnaire's content and the results of the questionnaire. Concerning occupation and income, the data showed that most patients have the ability to earn money. Our findings showed that the health system should pay greater attention to prevent TB among DM patients. The increased risk of active TB in DM patients should receive greater attention. In order to have early diagnosis and treatment, they should be informed to go to the hospital for examination in time when suspicious TB symptoms occur.

Table 1The demographic and anthropometric and lipid characteristics data of the study participants

\begin{tabular}{|c|c|c|c|}
\hline Demographic characteristics & $\begin{array}{c}\text { TB group } \\
(\mathrm{N}=81)\end{array}$ & $\begin{array}{l}\text { TB-DM group } \\
(\mathrm{N}=50)\end{array}$ & p-value \\
\hline Male, $n(\%)^{a}$ & $50(61.7)$ & $33(66.0)$ & 0.622 \\
\hline Age, (years),Mean $\pm S^{b}$ & $33.41 \pm 14.13$ & $48.60 \pm 13.48$ & 0.000 \\
\hline BMI, $\left(\mathrm{kg} / \mathrm{m}^{2}\right)$, Mean $\pm \mathrm{SD}^{\mathrm{b}}$ & $20.39 \pm 2.51$ & $22.14 \pm 3.07$ & 0.001 \\
\hline Education level, n(\%) ${ }^{\mathrm{a}}$ & & & 0.000 \\
\hline Primary school & $4(4.9)$ & $12(24.0)$ & \\
\hline Junior middle school & $16(19.8)$ & $19(38.0)$ & \\
\hline Senior middle school & $35(43.2)$ & $16(32.0)$ & \\
\hline Junior college & $15(18.5)$ & $1(2.0)$ & \\
\hline University or higher & 11(13.6) & $2(4.0)$ & \\
\hline Occupation, $\mathrm{n}(\%)^{\mathrm{a}}$ & & & 0.000 \\
\hline Farmer & $2(2.5)$ & $16(32.0)$ & \\
\hline Worker & $19(23.5)$ & $4(8.0)$ & \\
\hline Student & $14(17.3)$ & $2(4.0)$ & \\
\hline Leadership & $1(1.2)$ & 0 & \\
\hline Merchant & $3(3.7)$ & $2(4.0)$ & \\
\hline Retirement & $4(4.9)$ & $4(8.0)$ & \\
\hline Job-waiting & $17(21.0)$ & $13(26.0)$ & \\
\hline Migrant worker & 0 & $1(2.0)$ & \\
\hline Others & 21(25.9) & $8(16.0)$ & \\
\hline Average monthly income, $\mathrm{n}(\%)^{\mathrm{a}}$ & & & 0.035 \\
\hline$<1000$ & $36(44.4)$ & $15(30.0)$ & \\
\hline
\end{tabular}




\begin{tabular}{cccc}
\hline $1000-3000$ & $30(37.0)$ & $30(60.0)$ & \\
$3000-5000$ & $15(18.5)$ & $5(10.0)$ & 0.003 \\
Marital Status, $\mathrm{n}(\%)^{\mathrm{a}}$ & & & \\
Single & $35(43.2)$ & $9(18.0)$ & \\
Married & $46(56.8)$ & $37(74.0)$ & \\
Widowed & 0 & $1(2.0)$ & \\
Divorced & 0 & $3(6.0)$ & 0.005 \\
Smokers, $\mathrm{n}(\%)^{\mathrm{a}}$ & $51(63.0 \%)$ & $19(38.0 \%)$ & 0.026 \\
\hline Alcohol drinkers, $\mathrm{n}(\%)^{\mathrm{a}}$ & $47(58.0 \%)$ & $19(62.0 \%)$ & \\
\hline
\end{tabular}

a: chi- squared test ; b: t-test ; c: Mann-Whitney U test.

Food Diversification Levels and Nutrients Intake of Study population. The results of DDS showed that there were $86.4 \%$ of patients in TB patients group and $98 \%$ of patients in TB-DM patients group get the scores between 7 to 9 points, in which 9 points in the TB patients group and TB-DM patients group is $40.7 \%$ and $52 \%$ of the total number, respectively. There were $13.6 \%$ of TB patients group and $2 \%$ of TB-DM patients group less than 7 points, which means they did not meet dietary diversification (refer with: Table 2).

In these two groups, the intake of grains, fats and oils achieved the highest consumption rate, reaching $100 \%$. The types of food intake results show that intake of fish and shrimp, beans and its products, milk and dairy products are insufficient in TB patients group. And the consumption rate of milk and dairy products and beans and its products in TB patients group were obviously lower than that in TB-DM patients group ( $P=0.014 ; P=0.002)$. (refer with: Table3)

TB is regarded as a kind of malnutrition-related disease [13], dietary nutrition can also affect its occurrence and development to some degree besides effective drug treatment. Therefore, to improve the level of nutrition is an important component of comprehensive treatment. Some studies have found that the level of dietary diversification positively correlated with sufficient state of nutrient intake, which can effectively mirror the nutritional quality of diet[14, 15], and was a predictor of nutrient adequacy of the decisive factor[16]. It was obvious that supplementary state of nutrients in high dietary diversity level group was better than that of low level group [14, 17]. The World Health Organization and the United States Dietary Guidelines advised that people could increase their dietary nutritional quality though dietary diversification $[18,19]$.

This survey found that most members in TB-DM patients group could achieve dietary diversification, which may have an association with patient's income level. It seems that people in TB-DM patients group eat more beans and milk than that people in TB patients group, according to the results of the types of food intake. This situation may also have an association with patient's income level.

The results of Energy and Nutrients analyzed from 24h dietary recall data(Table4 ) showed that TB-DM patients' daily energy , protein, dietary fiber intake was $1626.02 \pm 506.94 \mathrm{kcal}, 67.11 \pm 22.18$ $\mathrm{g}$ and $12.25 \pm 6.58 \mathrm{~g}$, which were significantly lower than RNI $(p<0.05)$.Except Vitamin E, Iron, Phosphorus, Selenium and Manganese daily intake reached RNI, the rest of all kinds of vitamins and minerals dietary daily intake were lower than the $\mathrm{RNI}(p<0.05)$, Which means TB-DM patients' daily dietary intakes of nutrients were clearly insufficient in this study.

Our study showed that daily intake of energy and most nutrients of TB-DM patients in our study were significantly lower than the RNI. It was reported that Vitamin A played an important role in maintaining the structure of the alveolar epithelium, keeping the immune system's normal function , and enhancing the body's resistance to infection of the respiratory tract[20]. Calcium takes part in a variety of physiological activity of human body, because of the calcium deficiency in patients with pulmonary tuberculosis; decreased immunity and infection are more likely to occur. What's more, tuberculosis foci calcification requires large amount of Calcium, Calcium deficiency will go against the calcification process, and the illness delayed healing, as a result. Ascorbic Acid can regulate the function of the nervous system to reduce vascular infiltration, according to the results of clinical 
research, Ascorbic acid may have a significant therapeutic effect to exudative TB patients[21]. Riboflavin participated in energy metabolism and biological oxidation of human body. When body lacks Riboflavin, it may influence the body's biological oxidation, leading to metabolism disorders. Because of the storage of Riboflavin is rather limited, we should supplement it through our daily diet. The shortage of nutrients intake could lead to damage to numbers' of physiological function of the TB patients' body, as a result, the patient's condition got worse, and that is harmful for clinical treatment.

Table 2 DDS Ratings Distribution of the study participants

\begin{tabular}{cccc}
\hline DDS score & $\begin{array}{c}\text { TB group } \\
\mathrm{N}(\%)\end{array}$ & $\begin{array}{c}\text { TB-DM group } \\
\mathrm{N}(\%)\end{array}$ & $p$-value \\
\hline $1-6$ & $11(13.6)$ & $1(2)$ & 0.0256 \\
7 & $16(19.8)$ & $5(10)$ & 0.1394 \\
8 & $21(25.9)$ & $18(36)$ & 0.2206 \\
9 & $33(40.7)$ & $26(52)$ & 0.2493 \\
Total & $81(100)$ & $50(100)$ & \\
\hline
\end{tabular}

Table 3 Nine Categories of Food Consumption

\begin{tabular}{cccc}
\hline Types of food & TB group & TB-DM group & $p$-value \\
& N (\%) & N (\%) & - \\
\hline Grains & $81(100)$ & $50(100)$ & 0.862 \\
Vegetables & $79(97.5)$ & $49(98)$ & 0.423 \\
Fruits & $76(93.8)$ & $45(90)$ & 0.267 \\
Poultry meat & $76(93.8)$ & $49(98)$ & 0.165 \\
Fish and shrimp & $54(66.7)$ & $39(78)$ & 0.591 \\
Eggs & $76(93.8)$ & $48(96)$ & 0.014 \\
Milk and dairy products & $54(66.7)$ & $43(86)$ & 0.002 \\
Beans and its products & $56(69.1)$ & $46(92)$ & - \\
Fats and oils & $81(100)$ & $50(100)$ &
\end{tabular}

Table 4 Energy and Nutrients Intake Level of TB-DM patients group (Standard man-days *)

\begin{tabular}{|c|c|c|c|}
\hline Types of nutritions & $\begin{array}{l}\text { TB-DM group } \\
\quad(\mathrm{N}=50)\end{array}$ & RNI & p-value \\
\hline Energy [Kcal] & $1626.02 \pm 506.94$ & 2400 & 0.000 \\
\hline Protein [g] & $67.11 \pm 22.18$ & 75 & 0.015 \\
\hline Fat [g] & $49.32 \pm 24.26$ & - & - \\
\hline Dietary fiber [g] & $12.25 \pm 6.58$ & 30 & 0.000 \\
\hline Carbohydrate [g] & $228.48 \pm 84.16$ & - & - \\
\hline Retinol $[\mu \mathrm{g}]$ & $446.76 \pm 240.28$ & 800 & 0.000 \\
\hline Thiamine [mg] & $0.93 \pm 0.349$ & 1.4 & 0.000 \\
\hline Riboflavin [mg] & $0.95 \pm 0.30$ & 1.4 & 0.000 \\
\hline Niacin [mg] & $12.47 \pm 4.77$ & 14 & 0.028 \\
\hline Vitamin C [mg] & $81.18 \pm 44.98$ & 100 & 0.005 \\
\hline Vitamin E [mg] & $12.48 \pm 6.98$ & 10 & 0.015 \\
\hline Potassium [mg] & $1671.09 \pm 515.29$ & 2000 & 0.000 \\
\hline Sodium [mg] & $758.66 \pm 396.16$ & 2200 & 0.000 \\
\hline Calcium [mg] & $384.27 \pm 176.84$ & 800 & 0.000 \\
\hline
\end{tabular}




\begin{tabular}{cccc} 
Magnesium [mg] & $295.34 \pm 117.93$ & 350 & 0.002 \\
Iron [mg] & $18.53 \pm 9.68$ & 15 & 0.013 \\
Manganese [mg] & $4.89 \pm 1.97$ & 3.5 & 0.000 \\
Zinc [mg] & $9.68 \pm 3.92$ & 15.5 & 0.000 \\
Copper [mg] & $1.65 \pm 0.94$ & 2 & 0.010 \\
Phosphorus [mg] & $1045.30 \pm 319.59$ & 700 & 0.000 \\
Selenium [ $\mu \mathrm{g}]$ & $58.07 \pm 26.12$ & 50 & 0.034 \\
\hline
\end{tabular}

\section{Conclusion}

By the investigation, we found that TB-DM patients had a low daily intake of energy and a variety of nutrients, which may be related with local economic level and lack of knowledge. TB-DM patients were suggested to strengthen education of nutrition and health knowledge. In this process, we should pay attention to the old, low level of education and low family income groups. At the same time, we should adjust the diet structure, reduce influence of treatment caused by inadequate intake of nutrients, enhanced nutritional status of TB-DM patients and improve the body's immune function, enhanced disease resistance, and promote the speedy recovery of TB-DM patients.

\section{Acknowledgments}

The authors are grateful to all the staff in the TB services of Qingdao Chest Hospital for their help and coordination. We are grateful to all the participant in this study. This study was funded by the National Natural Science Foundation of China (NSFC, No.81472983) awarded to associate professor Wang Qiuzhen and National Natural Science Foundation of China (NSFC, No. 81172662) awarded to professor Ma Aiguo.

\section{References}

[1] Dooley, K. E. , R. E. Chaisson, "Tuberculosis and diabetes mellitus: convergence of two epidemics", J Lancet Infect Dis, 1 (2009) 737-46.

[2] Cheng, M. H., "Asia-Pacific faces diabetes challenge", J Lancet, 2 (2010) 2207-10.

[3] Jeon, C. Y. , M. B. Murray, "Diabetes mellitus increases the risk of active tuberculosis: a systematic review of 13 observational studies", J PLoS Med, 31 (2008) e152.

[4] Pablos-Mendez, A., J. Blustein , C. A. Knirsch, "The role of diabetes mellitus in the higher prevalence of tuberculosis among Hispanics", J Am J Public Health, 32 (1997) 574-9.

[5] Jick, S. S., E. S. Lieberman, M. U. Rahman , H. K. Choi, "Glucocorticoid use, other associated factors, and the risk of tuberculosis", J Arthritis Rheum, 34 (2006) 19-26.

[6] Kim, S. J., Y. P. Hong, W. J. Lew, S. C. Yang, E. G. Lee, "Incidence of pulmonary tuberculosis among diabetics", J Tuber Lung Dis, 33 (1995) 529-33.

[7] Wang, Q., A. Ma, I. C. Bygbjerg, X. Han, Y. Liu, S. Zhao , J. Cai, "Rationale and design of a randomized controlled trial of the effect of retinol and vitamin D supplementation on treatment in active pulmonary tuberculosis patients with diabetes", J BMC Infect Dis, 35 (2013) 104.

[8] GP, Z., "Discussion of Tuberculosis and Malnutrition", J Journal of Modern Medicine \& Health, 76 (2006) 1752-1759.

[9] Tan SY, C. H., Guan YH "The use of nutrition-assisting system in treating relapsing pulmonary tuberculosis", J Journal of Clinical Pulmonary Medicine, 91 (2005) 45-46. 
[10]WD, C., "Preparation and use of food-based dietary guidelines", J Food Nutrition and Agriculture, 82 (1997) 42-47.

[11]Wang YK, H. X., Wang QZ,, "Dietary Diversity Score and Nutrient Intakes of Tuberculosis Patients", J Food and Nutrition inChina, 97 (2011) 73-77.

[12] Leung, C. C., T. H. Lam, W. M. Chan, W. W. Yew, K. S. Ho, G. Leung, W. S. Law, C. M. Tam, C. K. Chan , K. C. Chang, "Lower risk of tuberculosis in obesity", J Arch Intern Med, 36 (2007) 1297-304.

[13] Y, L., "Tuberculosis and Nutrition", J Section of Respiratory SystemForeign Medical Sciences, 101 (2000) 110-112.

[14] Ogle BM, Hung PH , T. HT, "Significance of wild vegetables in micronutrient intakes of women in Vietnam: an analysis of food variety", J Antimicrob Agents Chemother, 86 (2001) 21-30.

[15] Hatloy A, Torheim LE , O. A, "Food variety-a good indicator of nutritional adequacy of the diet a case study from an urban area in Mali, West Africa.", J European Journal of Clinical Nutrition, 80 (1998) 891-898.

[16] Jin Y, Li YP , H. XQ, "Association Between Dietary Diversity and Nutrients Adeq uacy In Chinese Adult s", J Acta Nutrimenta Sinica, 109 (2009) 21-29.

[17] Murphy SP, Foote JA , W. LR, "Simple measures of dietary variety are associated with improved dietary quality.", J Journal of the American Dietetic Association, 110 (2006) 425-429.

[18] MT, R., "Operationalizing dietary diversity: A review of measure+ ment issues and research priorities", J The Journal of nutrition, 79 (2003) 3911-3926.

[19] Marshall TA, Stumbo PJ , W. JJ, "Inadequate nutrient intakes are common and are associated with low diet variety in rural community dwelling elderly", J The Journal of nutrition, 104 (2001) 2192-2196.

[20] Geng G, Luo ZX , F. Z, "The Progress in Correlation between Vitamin A and Lower Respiratory Tract Infections", J Journal of Pediatric Pharmacy, 77 (2009) 61-63.

[21] Xu P, Feng XH , Z. GM., "Further study on nutritional support for chronic tuberculosis", J Chinese Journal of Clinical Nutrition, 78 (1999) 170-177. 